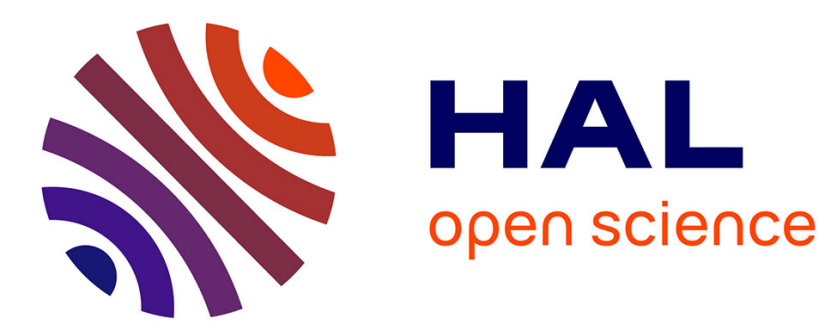

\title{
LA PURIFICATION DE L'ACIDE LACTIQUE PAR EXTRACTION
}

\author{
G. Génin
}

\section{To cite this version:}

G. Génin. LA PURIFICATION DE L'ACIDE LACTIQUE PAR EXTRACTION. Le Lait, 1956, 36 (351_352), pp.34-40. hal-00928156

\section{HAL Id: hal-00928156 https://hal.science/hal-00928156}

Submitted on 1 Jan 1956

HAL is a multi-disciplinary open access archive for the deposit and dissemination of scientific research documents, whether they are published or not. The documents may come from teaching and research institutions in France or abroad, or from public or private research centers.
L'archive ouverte pluridisciplinaire HAL, est destinée au dépôt et à la diffusion de documents scientifiques de niveau recherche, publiés ou non, émanant des établissements d'enseignement et de recherche français ou étrangers, des laboratoires publics ou privés. 
essais et nous avons utilisé un lait desséché par le même procédé (Spray) mais ayant subi un préchauffage.

\title{
RÉEERENCES BIBLIOGRAPHIQUES
}

[1] N. J. Berridge et C. Woodward. A simple method for preparing crystalline rennin. Journal Dairy Res., 20, 255-257, 1953.

[2] N. J. Berridge. Communication personnelle, 1954.

[3] H. Hostettuer et J. Stein. Beitrag zum Vorgang der Labgerinnung und zur Frage der Labstarkebestimmung. Landw. Jb. Schweiz., 68, 291-368, 1954.

[4] N. J. Berridge. The purification and erystallization of rennin. Biochem. Journal, 39, 179-186, 1945.

[5] R. VARIN. Thèse, Berne 1951.

[6] P. ZAHLER. Thèse, Berne 1953.

\section{SUPPLEMENT TECHNIQLE}

\section{LA PURIFICATION DE L'ACIDE LACTIQUE PAR EXTRACTION}

\author{
par G. GÉNIN
}

Ingénieur E.P.C.

D'après NeEdLe et ARIEs [1], la consommation annuelle d'acide lactique pourrait s'élever à plusieurs centaines de milliers de tonnes par an, si le prix de l'acide pur pouvait être réduit de façon sensible. Cet acide trouverait en particulier d'importants débouchés dans la fabrication des résines alkydes, des résines acryliques et d'autres résines. La purification de l'acide brut a toujours représenté une part importante du prix de revient de ce produit et il n'est done pas étonnant que d'importants travaux aient été entrepris sur ce point.

Nous avons mentionné, dans ces colonnés, différents procédés qui ont été proposés pour la purification de l'acide et nous rappellerons en particulier l'estérification de l'acide lactique brut en solution et la séparation de l'ester [2]. On a également proposé, comme procédé de purification possible, l'extraction liquide-liquide [3]. Dans un brevet, WaIte [4] a décrit l'extraction de l'acide par l'alcool amylique. On trouve enfin d'autres procédés dans diverses publications qui traitent de l'épuration de l'acide lactique et des propriétés physiques du produit obtenu [5].

Les principales impuretés que l'on trouve dans les solutions brutes d'acide lactique sont des sels de métaux alcalino-terreux, des sucres, et des impuretés volatiles telles que les acides acétique et butyrique [6]. La séparation de l'acide lactique de ces impuretés 
se trouve compliquée par la formation d'un polymère de l'acide lactique qui se produit très facilement, dès que la concentration en acide s'élève au-dessus de $20 \%$.

Le procédé de purification par extraction liquide-liquide paraissant plus particulièrement intéressant, R. B. WEISER et C. J. Geankoplis, de l'Ohio State University, à qui nous empruntons les renseignements qui suivent [7] ont procédé à une étude d'ensemble afin d'établir le mode de distribution de l'acide lactique et de diverses impuretés entre l'eau et un grand nombre de solvants organiques. Il a été ainsi possible de déterminer le solvant sélectif le meilleur, et on a examiné ensuite les possibilités d'emploi dans l'industrie de ce solvant.

\section{Matières premières utilisées dans la recherche}

Cette étude a porté sur de l'acide lactique chimiquement pur, dans certains cas, on a également employé de l'acide lactique du commerce à $22 \%$ de concentration. Tous les produits ajoutés à l'acide au titre d'impuretés, et les solvants utilisés étaient également des produits de grande pureté.

Dans tous les cas où cela a été possible, les dosages ont été effectués par des méthodes colorimétriques. Lorsque la concentration de l'acide lactique dans la solution aqueuse dépassait $20 \%$, le dosage de eet acide se trouvait compliqué par la présence de son polymère [8]. Dans ce cas, on ajoutait un excès de base à la solution et le mélange était chauffé pendant environ 10 minutes dans un bain d'eau bouillante. Pendant cette opération le flacon était bouché avec simplement présence d'un léger tube de dégagement évitant toute absorption du gaz carbonique de l'air par la solution. L'excès de base était ensuite filtré au moyen d'une solution étalonnée d'acide sulfurique.

Lorsque la solution d'acide comportait d'autres acides présents comme impuretés, le dosage de l'acide lactique s'effectuait par la méthode de Friedemann et Grasser [9]. Dans ce procédé, l'acide lactique est oxydé et transformé en acétaldéhyde et on forme le composé d'addition de cet aldéhyde et du bisulfite. La quantité de bisulfite est alors déterminée au moyen d'une solution étalon d'iode et on peut apprécier ainsi la teneur en acide lactique. On trouvera d'ailleurs dans l'original quelques autres indications concernant le dosage des autres substances susceptibles d'être ajoutées aux solutions d'acide lactique étudiées et en particulier les sucres.

Au cours des essais préliminaires, on a opéré sur des solutions renfermant $5 \%$ en poids d'acide lactique. On agitait $50 \mathrm{~cm}^{3}$ de cette solution et $50 \mathrm{~cm}^{3}$ de solvant, la température du mélange étant 
soigneusement contrôlée en plaçant le récipient dans un bain à température constante réglée à $\pm 0^{\circ} 05$ lorsqu'on opérait à $25^{\circ} \mathrm{C}$. et à $\pm 0^{\circ} 1$ lorsqu'on opérait à des températures plus élevées. La durée d'agitation nécessaire pour obtenir l'équilibre était déterminée en procédant à des analyses périodiques et dans tous les cas, la durée d'agitation était prise égale ou double du temps nécessaire à l'établissement de l'état d'équilibre. Dans les cas où la solution d'acide lactique était additionnée d'impuretés constituées par des sels organiques, le mode opératoire était le même.

On trouvera dans l'original de cette étude la liste des différents solvants étudiés en vue d'extraire l'acide lactique de ses solutions, et l'influence sur le coefficient de répartition de l'acide entre l'eau et le solvant d'un certain nombre d'additions, comme par exemple chlorure de sodium, sulfate de sodium, acide chlorhydrique, chlorure de calcium, ou des sucres tels que sucrose, lactose, ou enfin acide acétique, acide butyrique.

Le manque de place ne nous permet pas de reproduire ici la totalité des résultats numériques obtenus par WEISER et GEANkoplis. Nous nous contenterons de résumer les conclusions que l'on peut tirer de leurs résultats.

On a tout d'abord constaté que, si l'on groupe les différents solvants par analogie chimique, c'est-à-dire si on examine les solvants renfermant les mêmes groupes fonctionnels, on observe que si on porte sur une courbe tracée sur un papier logarithmique le coefficient de répartition de l'acide lactique entre eau et solvant, en fonction du poids moléculaire du solvant, on obtient une ligne droite. La ligne est évidemment différente suivant le groupe de solvants considéré, par exemple, suivant qu'il s'agit d'aleools primaires, secondaires ou tertiaires. On constate également, par l'examen des droites ainsi obtenues, que les méthylcétones sont de meilleurs solvants extractifs pour l'acide lactique que les eétones supérieures.

On a également étudié l'extraction de l'acide lactique en fonction de la température. Les courbes obtenues montrent qu'en général la température a peu d'effet sur l'extraction de l'acide par le solvant. On en a donc déduit que dans la pratique, la récupération de l'acide lactique pouvait s'effectuer à la température ordinaire.

On a également étudié l'extraction par le solvant de l'acide en fonction de la concentration de cet acide dans la solution aqueuse. On a constaté que pour les faibles concentrations auxquelles il faudra toujours opérer dans la pratique, l'influence de la concentration est faible.

En ce qui concerne enfin l'influence de certaines additions faites à la solution aqueuse, on a constaté que certains sels organi- 
ques favorisent l'extraction de l'acide lactique par les solvants. Cependant, le gain est relativement faible et ne justifie pas dans la pratique l'addition volontaire de sels à la solution à extraire.

La comparaison des coefficients de répartition de l'acide lactique entre la solution aqueuse et le solvant montre que d'une façon générale, ce sont les alcools qui constituent les meilleurs solvants extractifs de l'acide lactique. En outre, parmi ces alcools, les plus intéressants sont, à priori, les alcools inférieurs qui possèdent les propriétés physiques désirables et dont le prix est relativement bas. Parmi ces alcools, l'alcool isoamylique a l'avantage d'extraire de la solution aqueuse une faible quantité de sucre qui constitue l'impureté la plus fréquente dans les solutions d'acide lactique du commerce. Il a donc été décidé de procéder à un examen plus approfondi de l'extraction de l'acide lactique de ses solutions aqueuses, par l'alcool isoamylique.

\section{Extraction de l'acide lactique par l'alcool isoamylique}

Cette étude a été entreprise au moyen d'alcool isoamylique chimiquement pur ayant subi cependant un traitement complémentaire de purification par distillation dans une colonne de laboratoire. On a ainsi pu obtenir un produit bouillant entre 131,5 et $132^{\circ}$, présentant un indice de réfraction de 1,4085 à $15^{\circ}$ et possédant, par conséquent, les caractéristiques physiques de l'alcool isoamylique pur [10].

Le tableau ci-dessous indique le coefficient de distribution de l'acide lactique entre l'eau et l'alcool isoamylique et l'influence, sur ce oefficient, d'un certain nombre de facteurs tels que température, concentration de l'acide dans la solution aqueuse, et présence de différents produits d'addition.

On a opéré dans certains cas, non seulement sur l'alcool distillé ou non distillé, comme il a été indiqué précédemment, mais également sur l'acide lactique chimiquement pur du commerce, ainsi que sur l'acide lactique brut et non purifié.

\section{COEFFICIENT DE DISTRIBUTION DE L'AGIDE LACTIQUE ENTRE L'EAU ET L'ALCOOL ISOAMYLIQUE.}

$\mathrm{Cw}=$ concentration $\mathrm{du}$ corps dissous dans la phase aqueuse, $\mathrm{g} / 100 \mathrm{~cm}^{3}$.

$\mathrm{Cs}=$ concentration $\mathrm{du}$ corps dissous dans la phase organique, $\mathrm{g} / 100 \mathrm{~cm}^{3}$. $\mathrm{K}=\mathrm{Cs} / \mathrm{Cw}$.

Température Degré C. Produit d'addition 


$\begin{array}{cccc}\text { Température Degré C. } & \text { Produit d'addition } & \mathrm{Cw} & \mathrm{K} \\ 25 & & 11,93 \mathrm{~b} & 0,487 \mathrm{a} \\ 25 & & 16,24 \mathrm{~b} & 0,509 \mathrm{a} \\ 25 & & 22,38 \mathrm{~b} & 0,563 \mathrm{a} \\ 38,9 & & 4,07 \mathrm{~b} & 0,479 \\ 49,5 & & 3,99 \mathrm{~b} & 0,505 \\ 58,5 & 3,91 \mathrm{~b} & 0,527 \\ 25 & 5 \% \mathrm{HCl} & 1,99 \mathrm{~b} & 0,545 \\ 25 & 5 \% \mathrm{NaCl} & 4,12 \mathrm{~b} & 0,480 \\ 25 & 20 \% \mathrm{NaCl} & 3,22 \mathrm{~b} & 0,686 \\ 25 & 5 \% \mathrm{SO}^{4} \mathrm{Na}^{2} & 4,07 \mathrm{~b} & 0,462 \\ 25 & & 4,26 \mathrm{c} & 0,442 \mathrm{a} \\ 25 & & 5,64 \mathrm{c} & 0,452 \mathrm{a} \\ 25 & & 8,41 \mathrm{c} & 0,470 \mathrm{a} \\ 25 & & 16,51 \mathrm{c} & 0,521 \mathrm{a}\end{array}$

a : alcool purifié par distillation.

b : acide lactique chimiquement pur.

c : acide lactique brut.

En utilisant ce tableau, il est possible par exemple, d'établir l'influence de la concentration sur le coefficient de distribution de l'acide lactique entre l'eau et l'alcool isoamylique, aussi bien dans le cas de l'acide lactique pur que dans le cas de l'acide lactique du commerce. Si on admet que l'impureté acide est entièrement sous forme d'acide acétique, et si on tient compte de la présence de cet acide, on constate que la courbe représentant le coefficient de distribution en fonction de la concentration est pratiquement la même, qu'il s'agisse d'acide lactique pur ou d'acide lactique du commerce.

On a également étudié avec les résultats numériques reproduits dans le tableau, la variation de la solubilité de l'alcool isoamylique dans les solutions aqueuses d'acide lactique. On constate que cette solubilité reste faible, lorsque la concentration en acide lactique reste de l'ordre de grandeur de celle des solutions d'acide brut qu'on aurait pratiquement à traiter. On peut donc admettre que la perte de solvant dans une colonne d'extraction ne serait pas importante.

\section{Etude d'une installation industrielle de récupération de l'acide lactique}

Pour qu'une installation de récupération d'un corps dissous par extraction liquide-liquide fonctionne d'une façon satisfaisante, il n'est pas seulement nécessaire que le solvant ait les propriétés de sélectivité voulues, encore faut-il que d'autres conditions soient remplies et que par exemple la récupération du solvant et celle du produit qu'il a dissous soit réalisable dans des conditions relati- 
vement simples. Pour récupérer le solvant, on dispose de deux méthodes qui sont l'entraînement par la vapeur d'eau ou une extraction inverse. L'entraînement par la vapeur d'eau présente l'avantage que cette opération peut éliminer de la solution d'acide lactique, non seulement le solvant, mais les impuretés acides volatiles et on obtient dans ces conditions une solution aqueuse d'acide lactique relativement pure et en particulier privée d'acide volatil. On peut ensuite concentrer la solution d'acide lactique ainsi purifiée par distillation avec emploi d'un agent d'entraînement.

Ce sont ces différents points qui ont été examinés par WErsER et J. GEankoplis qui ont opéré dans ce but dans une installation de laboratoire constituée par un ballon d'une capacité de 1 litre, dans lequel on faisait passer un courant de vapeur d'eau destiné à entraîner l'alcool isoamylique et les acides volatils. L'essai a été fait en opérant sur un mélange préparé spécialement d'eau, d'acides acétique et butyrique, ainsi que sur un mélange obtenu par extraction au moyen d'alcool isoamylique, d'une solution brute d'acide lactique du commerce.

Cette étude a montré que l'on peut par exemple, au cours de cette opération, éliminer la totalité des acides volatils contenus dans la solution traitée, sans perdre d'acide lactique. On en a effet obtenu au cours de plusieurs essais successifs des rendements en acide lactique atteignant $99,6 \%$.

En ce qui concerne la consommation de vapeur d'eau, les essais effectués en laboratoire ont conduit à une consommation de vapeur par rapport au poids d'alcool isoamylique à extraire de $7 \mathrm{~kg} .9$ de vapeur pour 1 kilogramme d'alcool isoamylique. Il est certain que dans une installation industrielle, cette consommation pourrait être considérablement réduite.

On trouvera dans l'original le schéma d'une installation industrielle imaginé par les auteurs, en tenant compte des résultats de leurs études en laboratoire. L'installation comporte en particulier une colonne d'extraction fonctionnant en contre-courant, ainsi qu'un appareil de concentration dans le vide pour la concentration de la solution d'acide lactique obtenue, après récupération du solvant.

L'expérience a montré que la solution d'acide lactique qu'on obtient par ce procédé est toujours légèrement colorée, mais il est facile de la décolorer en la faisant passer sur un lit de charbon activé et dans ce cas, la perte d'acide est d'environ $1 \%$. Il semble donc que le procédé d'extraction de solutions brutes d'acide lactique par l'alcool isoamylique présente un intérêt certain, il sera cependant nécessaire de confirmer cette opinion par des essais semi-industriels portant sur une installation pilote. G. Génin. 


\section{RÉFÉRENCES}

[1] H. C. NeEdLe et R. S. Aries. Sugar, 1949, 44, 32.

[2] A. A. Dietz, E. F. Degering et H. H. Schopmeyer. Ind. Eng. Chem., $1947,39,82$.

E. M. Filachione et C. H. Fisher. Ind. Eng. Chem., 1946, 38, 228.

E. M. Filachione et C. H. Fisher. Br. am. 2.420.234, 6 mai 1947.

E. M. Filachione, J. H. Lengel et C. H. Fisher. Ind. Eng. Chem., $1945,37,388$.

[3] J. A. JenEMANN. Br. am. 1.906.068, 25 avril 1933.

R. H. Leonari, W. H. Peterson et N. J. Johnson. Ind. Eng. Chem., 1948, 40, 57.

R. Montgomery et R. A. Ronca. Ind. Eng. Chem., 1953, 45, 1136.

W. P. Ratchford, E. H. Harris, C. H. Fisher et C. O. Willits. Ind. Eng. Chem., 1951, 43, 778.

L. T. Вмгтн et H. V. Cla.born. Ind. Eng. Chem., News Ed., 1939, 17, 641.

[4] C. N. WaIte. Br. am. 686.170, novembre 1901.

[5] E. M. Filachione et C. H. Fisher. Ind. Eng. Chem., 1944, 36, 223.

G. T. Peckнам. Chem. Eng. News, 1944, 22, 440.

P. D. Watson. Ind. Eng. Chem., 1940, 32, 399.

[6] S. L. BAss. Br. am. 2.092,494. 7 septembre 1937.

[7] R. B. Weiser et C. J. Geankoplis. Ind. Eng. Chem., 1955, 47, 858.

[8] R. Eder et F. Kutwer. Helv. Chim. Acta, 1926, 9, 557.

A. R. Hickinbotham. Analyst, 1948, 73, 509.

[9] T. E. Friedemann et J.B. Graeser. Journal Biol. Chem., 1933, 100, 291.

[10] Handbook of Chemistry, publié par Handbook Publishers, Sandusky, 1949,7 e édition, 941.

\section{Bulletin analytique}

\section{Revues}

\section{Textiles}

Orsini (A.). - Fibres de protéines régénérées. Industrie textile, n 0807, p. $87,1954$.

Description de la composition, des propriétés et de la fabrication des fibres de caséine, de zéine et de protéines d'arachides.

G. Génin.

\section{Frolich (H.G.). - Teinture VI. L'absorption des colorants acides et des colorants au chrome par les fibres de protéines modifiées. $Z$, ges. Textil-Ind. 1953, t. 55, p. 1267.}

Etude de l'absorption des colorants par les fibres de protéine normales, acétylées et déaminées, dans le cas du colorant au chrome (Palatin) et influence sur cette absorption du $p \mathrm{H}$ et de la concentration en colorant de la solution. 